\title{
New horizons in multidimensional diffusion: The Lorentz gas and the Riemann Hypothesis
}

\author{
Carl P. Dettmann, School of Mathematics, University of Bristol, Bristol BS81TW, UK
}

November 10, 2018

\begin{abstract}
The Lorentz gas is a billiard model involving a point particle diffusing deterministically in a periodic array of convex scatterers. In the two dimensional finite horizon case, in which all trajectories involve collisions with the scatterers, displacements scaled by the usual diffusive factor $\sqrt{t}$ are normally distributed, as shown by Bunimovich and Sinai in 1981. In the infinite horizon case, motion is superdiffusive, however the normal distribution is recovered when scaling by $\sqrt{t \ln t}$, with an explicit formula for its variance. Here we explore the infinite horizon case in arbitrary dimensions, giving explicit formulas for the mean square displacement, arguing that it differs from the variance of the limiting distribution, making connections with the Riemann Hypothesis in the small scatterer limit, and providing evidence for a critical dimension $d=6$ beyond which correlation decay exhibits fractional powers. The results are conditional on a number of conjectures, and are corroborated by numerical simulations in up to ten dimensions.
\end{abstract}

\section{Introduction}

The Lorentz gas is a billiard model [13, in which a point particle moves in straight lines with unit speed except for specular collisions with hard scatterers, most often disks or balls. It is an "extended" billiard in that it moves in an unbounded domain, in contrast to the usual billiard setting. Lorentz developed an approach along these lines to study electrical conduction in 1905 [29], however periodic arrays of scatterers in the plane or in higher dimensions appeared as early as 1873 (the Galton board, or "quincunx" [21]). They have been a major example of hyperbolic dynamics with singularities starting from the work of Sinai in 1970 42.

Lorentz gas models are considered in the physics literature, for example arising as the molecular dynamics model with two atoms and a hard potential after reduction of the centre of mass motion [17. It is thus probably the simplest model of deterministic diffusion with physical attributes such as a Hamiltonian structure, continuous time dynamics and time reversal invariance; simpler but less physical models include one dimensional maps on $f: \mathbb{R} \rightarrow \mathbb{R}$ with a periodicity property $f(x+1)=f(x)+1$ [20, 39] and multibaker maps on $[0,1]^{2} \times \mathbb{Z}[22$. In the former case, with $f$ a piecewise linear map, the diffusion constant is a non-smooth function of parameters, as quantified in [26]. It would be interesting to extend such results to billiards; numerical studies of Lorentz(-like) models have suggested an irregular but somewhat smoother behaviour than the piecewise linear map [24, 25, 27, 28,

There is a substantial literature on diffusion in periodic Lorentz gases in dimension $d=2$ in the setting of dispersing billiards, for example sufficiently smooth $\left(C^{3}\right)$, convex with curvature bounded away from zero, and an upper bound on the free flight length between scatterers ('finite horizon'). It is known that the displacements of particles initially distributed uniformly (outside the scatterers) converges weakly to a Wiener process (Brownian motion) with variance proportional to the (continuous) time $t$ as $t \rightarrow \infty$ [7]; proof of recurrence in the full space followed later [15, 40. The strategy is usually to relate the deterministic model to a Markovian random walk process for which the results are known or easier to prove.

If we relax the finite horizon condition, the distribution remains Gaussian, but with a $t \ln t$ scaling. The two dimensional case was considered by Zacherl et al. [46] who gave approximate formulas for the velocity correlation functions, and hence diffusion properties. Bleher [5], proved some of the necessary results, deriving 
some exact coefficients. Detailed numerical results for velocity correlations were obtained by Matsuoka and Martin [32. Szász and Varju [45, completed the proofs in discrete time $(n)$, obtaining a local limit law to a normal distribution, and also recurrence and ergodicity in the full space. Techniques there were based on work of Bálint and Gouëzel [2] for the stadium billiard, which also has long flights without a collision on a curved boundary, and also a normal distribution with a nonstandard $n \ln n$ limit law. Finally, Chernov and Dolgopyat [1] proved weak convergence to a Wiener distribution in the continuous time case.

In general it is easier to prove statements about correlation decay and diffusion for the discrete time dynamics first; some diffusion results can then be transferred to continuous time, as discussed in Ref. [5, 11] and Section 9 below. Correlation decay for billiards in continuous time have been considered [3, 9, 33, but are in general more difficult. Here we use continuous time $(t)$ with connections to the discrete time diffusion process discussed in Sec. 9 .

The main purpose of this paper is to explore the infinite horizon case in dimensions $d>2$, or more precisely, several varieties of infinite horizon that appear in higher dimensions. Recurrence is of course not to be expected, even for finite horizon, however a (super)-diffusion coefficient and further refinements may still be considered. One case in which the diffusion in higher dimensional Lorentz gases has been treated very recently is the Boltzmann-Grad limit [30, 31]. Here the radius and density of scatterers scale so that the mean free path is constant. Here we will also consider the limit of small scatterers, but only after the infinite time limit. We will nevertheless be able to make connection with an asymptotic formula in 31. In addition, we will find connections with the Riemann Hypothesis, along the lines of those presented for the escape problem of a billiard inside a circle with one or two holes [ 6 ; this is also new for $d=2$. Last but not least, there are some outstanding issues in $d=2$ regarding the diffusion coefficients defined using moments and using a limiting normal distribution, which we need to clarify first (Sec. 20; these may also have wider implications for our understanding of other anomalous diffusion processes. Main results are stated and discussed in Sec. 3, which concludes with a description of the following more detailed sections.

\section{Diffusion coefficients}

Different conventions are used in physical and mathematical literature for diffusion, and there are further subtleties involved with the definition of the logarithmic superdiffusion coefficient. Fick's law, dating back to 1855 , states that the flux of particles in a diffusing species is proportional to the concentration gradient. If, as in the Lorentz gas, the diffusing particles do not interact with each other, and the medium through which they diffuse (periodic collection of scatterers) is homogeneous at large scales but not necessarily isotropic, from physical considerations we expect a macroscopic equation of the form

$$
\frac{\partial \rho}{\partial t}=D_{i j} \frac{\partial}{\partial \tilde{x}_{i}} \frac{\partial}{\partial \tilde{x}_{j}} \rho
$$

where $\rho(\tilde{\mathbf{x}}, t)$ is the density of particles, $i$ and $j$ are spatial indices, we are using the Einstein summation convention (summing repeated indices from 1 to $d$ ), and we denote quantities in the full (unbounded) space with tildes. The $D_{i j}$ are components of the diffusivity matrix $\mathbf{D}$, which is positive semi-definite and symmetric. For now we assume that it is actually positive definite, corresponding to the possibility of diffusion in all directions. In simple cases it is often isotropic, $D_{i j}=D \delta_{i j}$ where $D$ is "the" diffusion coefficient or diffusivity. The Green's function of Eq. (1) is a Gaussian

$$
\rho(\tilde{\mathbf{x}}, t)=\frac{e^{-\frac{D_{i j}^{-1} \tilde{x}_{i} \tilde{x}_{j}}{4 t}}}{\sqrt{4 \pi t \operatorname{det} \mathbf{D}}}
$$

involving the inverse matrix $\mathbf{D}^{-1}$. The second moment of position with respect to this density is

$$
\int \rho(\tilde{\mathbf{x}}, t) \tilde{x}_{i} \tilde{x}_{j} d \mathbf{x}=2 D_{i j} t
$$

The macroscopic picture may be connected to the microscopic dynamics in terms of the displacement of a single particle $\Delta(t)=\tilde{\mathbf{x}}(t)-\tilde{\mathbf{x}}(0)$. If we assume the microscopic dynamics is periodic with respect to a 
lattice of finite covolume, we can define a configuration variable $\mathbf{x}$ modulo lattice translations, and consider the dynamics as a random process defined using a probability measure $\mu$ on its initial position and velocity $(\mathbf{x}(0), \mathbf{v}(0))$. Expectations with respect to this measure will be denoted by angle brackets \langle\rangle . Depending on the details of the dynamics, it may be possible to prove that in the $t \rightarrow \infty$ limit $\Delta$ has the appropriate second moment

$$
\lim _{t \rightarrow \infty} \frac{\left\langle\Delta_{i} \Delta_{j}\right\rangle}{2 t}=D_{i j}
$$

and/or that it converges weakly to a normal distribution

$$
\frac{\Delta}{t} \Rightarrow \mathcal{N}\left(0, \Sigma_{i j}\right)
$$

with zero mean and covariance matrix $\Sigma_{i j}=2 D_{i j}$. Convergence of the process to Brownian motion (ie multiple time distributions) with the same covariance matrix has also been shown in some cases. Note in particular the factors of 2 . We may want an overall diffusivity, generalising the isotropic case:

$$
D=\frac{1}{d} \operatorname{tr} \mathbf{D}=\lim _{t \rightarrow \infty} \frac{1}{2 d t}\left\langle\Delta_{i} \Delta_{i}\right\rangle
$$

for example Bleher [5] uses this for $d=2$.

The dynamics may lead to anomalous diffusion, where the second moment may not be linear in $t$. Conventions differ as to how the diffusivity is generalised. Here we consider cases where the second moment is logarithmic. By analogy with the normal distribution case above we define

$$
\lim _{t \rightarrow \infty} \frac{\left\langle\Delta_{i} \Delta_{j}\right\rangle}{2 t \ln t}=\mathcal{D}_{i j}, \quad \mathcal{D}=\frac{1}{d} \operatorname{tr} \mathcal{D}_{i j}, \quad \frac{\Delta}{t \ln t} \Rightarrow \mathcal{N}\left(0, \Xi_{i j}\right)
$$

where the relevant limits exist, but now it is no longer clear whether $\mathcal{D}_{i j}$ is related to $\Xi_{i j}$, as noted in [16]. Convergence in distribution does not imply convergence of the moments, since the tails may decay slowly while spreading further with time; see Fig. 1. The authors of Ref. [16] numerically considered higher moments of $\Delta(n) / \sqrt{n \ln n}$ but it is likely that recent methods [35] will lead to a proof of an anomalous exponent without a logarithm for these higher moments and with a logarithm for lower moments [34. This proposed work does not include our case of interest (second moment) which is borderline between regimes of the normal and abnormal moments. We continue the discussion of this anomalous moment below.

As with the normal diffusion, we assume that superdiffusion is possible in all directions; in general it is possible to have normal diffusion in some directions and superdiffusion in others, as discussed in [45, 11]. Also, the definition for $\Xi$ makes sense with respect to discrete (collision) time $n$ replacing $t$; we expect the relation $\Xi^{\text {disc }}=\tau \Xi$ with $\tau$ the mean free time; see [5, 11] and Sec. 9. Note however that $\mathcal{D}^{\text {disc }}$ is problematic since $\left\langle\Delta^{2}\right\rangle$ is infinite for a single free path as well as $n$ free paths, and hence not susceptible to normalisation by any function of $n$.

Noting $\Delta_{i}=\int_{0}^{t} v_{i}(s) d s$ we find (see Eq. 1.4 in Ref. [5]) the expression

$$
\frac{1}{2}\left\langle\Delta_{i} \Delta_{j}\right\rangle=t \int_{0}^{t}\left\langle v_{i}(0) v_{j}(s)\right\rangle d s-\int_{0}^{t} s\left\langle v_{i}(0) v_{j}(s)\right\rangle d s
$$

which leads to the usual continuous time Green-Kubo formula for $D_{i j}$ in the limit $t \rightarrow \infty$, assuming the velocity autocorrelation function (VACF) decays sufficiently rapidly. If instead, we have $\left\langle v_{i}(0) v_{j}(s)\right\rangle \sim C_{i j} / s$, we find $\mathcal{D}_{i j}=C_{i j}$. See Ref. 33] for rigorous results on continuous time decay of correlations for this system. Following Bleher [5], we note that in this (continuous time) case, the superdiffusion arises from the slow decay of the VACF, but that in the discrete case, the correlations decay rapidly, but superdiffusion arises from the infinite second moment of the displacement between collisions.

We now return to the specific case of the periodic Lorentz gas and discuss existing results for $d=2$. The Lorentz gas is a billiard in an unbounded domain with a periodic collection of convex obstacles. If the free path length is bounded away from zero and infinity ("finite horizon"), the diffusion is normal, as noted in the Introduction. The Green-Kubo formula is valid, but there is no closed form expression for the correlation functions or diffusion coefficient. 


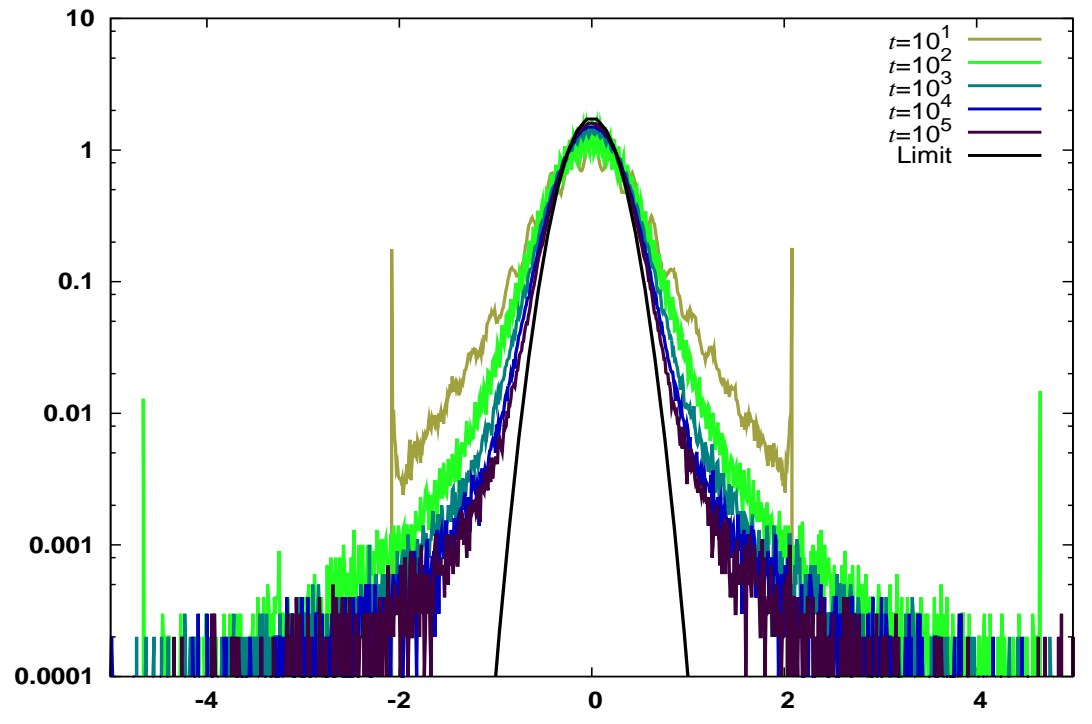

Figure 1: The distribution of the scaled displacement $\Delta / \sqrt{t \ln t}$ for the square $(d=2)$ Lorentz gas with $r=0.4$, showing convergence to the expected normal distribution, but with fat expanding tails at any finite time that support the argument in the text for an anomolously large second moment. Two features observed here at short times are oscillations due to the lattice, and spikes arising from ballistic orbits with no collisions that indicate the boundaries of the support of the distribution. 
In the infinite horizon case there are infinite strips that do not contain any scatterers, termed "corridors" that lead to logarithmic superdiffusion as described above. These corridors can be enumerated, leading to exact expressions for the superdiffusion coefficient. We assume that there are at least two non-parallel corridors, so that superdiffusion occurs in all directions.

For a comparison of the coefficients existing in the literature, we need only consider the specific case of a Lorentz gas with a square lattice of unit spacing and disks of radius $1 / \sqrt{8}<r<1 / 2$, for example the case $r=0.4$ considered in numerical simulations below. This has only corridors in the horizontal and vertical directions. Bleher [5] following Eq. 8.5 writes a discrete superdiffusion coefficient (in our notation)

$$
\mathcal{D}_{\text {Bleher }}^{\text {disc }}=\frac{w^{2}}{2 \pi r}
$$

where $w=1-2 r$ is the width of the corridor, and $2 \pi r$ is the circumference of a scatterer. As noted above the second moment of $\Delta$ is actually infinite. Bleher attempts to circumvent this issue by putting the $n \rightarrow \infty$ limit inside the average, but this also does not make sense at face value. If we make the interpretation $\mathcal{D}^{\text {disc }}=\tau \mathcal{D}$ it agrees with the calculations presented here.

Szasz and Varju ([45], Eq. (2)), and later Chernov and Dolgopyat ([11], Eq. (2.1)), give for the components of the covariance matrix

$$
\Xi_{i j}^{\mathrm{disc}}=\sum_{x} \frac{c_{\mu} w_{x}^{2}}{2|\psi(x)|} \psi_{i}(x) \psi_{j}(x)
$$

where $x$ is a fixed point of the collision map of the reduced (unit cell) space, assuming that each side of the corridor contains periodic copies of only one scatterer; there are four of these for each corridor. $c_{\mu}=1 /(4 \pi r)$ is the normalising factor for the invariant measure of this map, equal to half the reciprocal perimeter of the billiard. $w_{x}$ is the width of the corridor corresponding to $x$. Finally $\psi(x)$ is the vector parallel to the corridor giving the translation of $x$ under the map in the full space, with components $\psi_{i}(x)$. For the square lattice, $\psi(x)$ is a unit vector aligned with the coordinate axes, and taking account of the above mentioned factor of four, we find

$$
\Xi_{i j}^{\text {disc }}=\frac{w^{2}}{2 \pi r} \delta_{i j}
$$

Thus in contrast to the normal diffusion case it appears that $\Xi \neq 2 \mathcal{D}$, in fact $\Xi=\mathcal{D}$. This discrepancy is not noted in existing literature, presumably due to the different notations used. However, Balint, Chernov and Dolgopyat have reportedly proposed it for this (two dimensional) case, independently of and prior to the present author, and claim to have a proof [10.

From Eq. (8) we can (heuristically) explain the discrepancy as follows. Given a random initial condition (according to the usual continuous time invariant probability measure $\mu$ ), there is a probability $\sim C / t$ that it will make a free flight for at least a long time $t$. This is much higher than the exponentially small probability of travelling this distance according to the normal distribution. Thus the latter can be accurate at best only to distances

that is,

$$
\frac{e^{-x^{2} /(2 \Xi t \ln t)}}{\sqrt{2 \pi \Xi t \ln t}} \approx \frac{C}{x}
$$

$$
x \approx \sqrt{\Xi t \ln ^{2} t}
$$

Orbits dominated by a free flight much longer than this are well outside the converged part of the normal distribution and thus cannot contribute to its variance. Splitting the integrals in Eq. (8) into times less than, or greater than $\sqrt{t}$, we find that the first term gives a contribution to $\mathcal{D}$ from the orbits that could contribute to the normal distribution of $(C / 2) t \ln t$, that is, exactly half its full value (modulo lower order terms like $t \ln \ln t$ arising from the logarithm in Eq. (13)). Thus we expect $\Xi=\mathcal{D}$ in agreement with Eq. (11). Note that this argument depends on the specific mechanism for the slow decay of the VACF, and may not apply to all processes with logarithmic superdiffusion. It should however extend to the Lorentz gas in arbitrary dimension considered for the remainder of this paper.

Thus we propose that the second moment is anomalous, specifically by a factor of two, giving the equation $\Xi=\mathcal{D}$ in two and higher dimensions. In $d=2$ we agree with recent rigorous expression for $\Xi$ in Refs. [11, 45] and Bleher's $\mathcal{D}$. However, some of Bleher's discussion of $\mathcal{D}^{\text {disc }}$ and $\Xi$ appears problematic. 

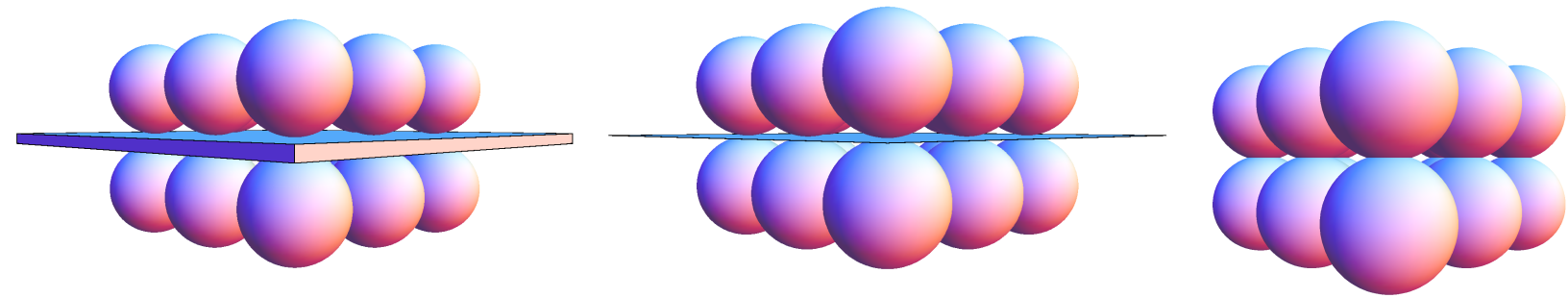

Figure 2: Horizons in the cubic Lorentz gas, depicted with a $3 \times 3 \times 2$ subset of the lattice, as viewed from one corner, for different scatterer radii, and illustrating the horizon terminology introduced in the text. If the scatterers are small enough that they don't touch $(r<0.5$, left $)$ there are planar horizons that are principal (and hence maximal) and not incipient. When the scatterers touch $(r=0.5$, centre), they create an incipient principal horizon. When the spheres overlap $(r>0.5$, right), cylindrical horizons are possible as seen in the figure; these are maximal but not principal.

\section{Summary of results}

As discussed previously for the case $d=2$ [5, 11, 45] superdiffusion in an infinite horizon Lorentz gas is a result of the unbounded path length of the particle, thus an understanding requires close study of the properties of these paths. Here we extend the concept of a horizon (or "channel") as it appears in existing literature. Usual terminology defines free paths as bounded from above ("finite horizon") or infinite ("infinite horizon") with occasional variations ("finite but unbounded", also called "locally finite", for some aperiodic Lorentz gases). Sanders 37] makes a finer classification of infinite horizon billiards in three dimensions using the dimensionality of the horizon (cylindrical or planar horizon billiards). Here we define a horizon, not as a type of bound or dimension, but as a set of points whose trajectories have no collisions with the scatterers, and satisfy some natural conditions.

Some definitions we need in this section (with more details in Sec. 4) are as follows. We consider the compact configuration space $M_{x} \subset \mathbb{R}^{d} / \mathcal{L}$ where the lattice $\mathcal{L}$ of covolume $\mathcal{V}$ is the discrete subgroup of $\mathbb{R}^{d}$ defining the periodicity of the Lorentz gas. The scatterers cover a volume fraction $\mathcal{P}=1-\left|M_{x}\right| / \mathcal{V}$. The continuous time dynamics $\Phi^{t}$ acts on the phase space $M=M_{x} \times M_{v}$ with $M_{v}=\mathbb{S}^{d-1}$, the set of velocities of unit magnitude. The set $M_{t} \subset M$ denotes initial conditions that do not collide with a scatterer for times in $[0, t]$. A horizon $H \subset M_{\infty}$ is of the form $H=H_{x} \times H_{v}$ with $H_{v}=\mathbb{S}^{d-1} \cap V_{H}$ and $V_{H} \subset \mathbb{R}^{d}$ is a linear space of dimension $d_{H} \in\{1 \ldots d-1\}$, the "dimension of the horizon." The set $H_{x}$ is connected and invariant under translations in $V_{H}$. Projecting onto the $d-d_{H}$ dimensional orthogonal space $V_{H}^{\perp}$ we obtain a lattice $\mathcal{L}_{H}^{\perp}$ of covolume $\mathcal{V}_{H}^{\perp}$ and a set characterising the horizon $H_{x}^{\perp}$. There is a natural invariant probability measure on $M$, denoted $\mu$. The probability of surviving without a collision for time $t$ is $F(t)=\mu\left(M_{t}\right)$, while the probability of remaining within a horizon $H$ (or more precisely its spatial projection) is

$$
F_{H}(t)=\mu\left(\left\{(\mathbf{x}, \mathbf{v}): \Phi^{s}(\mathbf{x}, \mathbf{v}) \in H_{x} \times M_{v}, \quad \forall s \in[0, t]\right\}\right) .
$$

We make further distinctions, illustrated in Fig. 2. A maximal horizon is one of highest dimension for the given billiard, a principal horizon is one of the highest dimension possible, $d-1$ (planar in Sanders' case) and an incipient horizon is one where $H_{x}^{\perp}$ has zero $d-d_{H}$ dimensional Lebesgue measure, hence $F_{H}(t)=0$ for all $t$. For example, a principal horizon has a connected one-dimensional set $H_{x}^{\perp}$ that is an interval, of length $w_{H}$, the "width" of the horizon. When this interval shrinks to a point, as by increasing the size of the scatterers, the horizon has scatterers touching it on both sides and becomes incipient. The set of maximal non-incipient horizons is denoted $\mathcal{H}$. Lemma 1 in Sec. 5 asserts that this set is finite.

We will see that the decay exponent of $F(t)$ is determined by the dimensionality of the maximal horizon with special conditions related to incipient horizons, and $\mathcal{D}_{i j}$ and $\mathcal{D}_{i j}^{\text {disc }}$ are nonzero only for non-incipient principal horizons. 


\begin{tabular}{ccccccc}
\hline \hline$d$ & 0 & 1 & 2 & 3 & 4 & general \\
\hline$S_{d}$ & 2 & $2 \pi$ & $4 \pi$ & $2 \pi^{2}$ & $\frac{8 \pi^{2}}{3}$ & $\frac{2 \pi^{(d+1) / 2}}{\Gamma((d+1) / 2)}$ \\
$V_{d}$ & 1 & 2 & $\pi$ & $\frac{4 \pi}{3}$ & $\frac{\pi^{2}}{2}$ & $\frac{\pi^{d / 2}}{\Gamma(d+2) / 2)}$ \\
$G_{d}$ & - & - & $\frac{1}{2 \pi}$ & $\frac{1}{4}$ & $\frac{1}{\pi}$ & $\frac{\Gamma(d / 2)}{2 \sqrt{\pi} \Gamma((d-1) / 2)}$ \\
\hline \hline
\end{tabular}

Table 1: Lebesgue measure of $d$-dimensional spheres, $S_{d}$ and balls $V_{d}$, and the combination $G_{d}=S_{d-2} / 2 S_{d-1}$ appearing in Eq. 27 below, including specific values of interest and the general formulae (last column).

Although much progress has been made on higher dimensional billiards in the last decade, some major challenges remain 44. Existing results are not sufficient to provide a fully unconditional foundation for the study of diffusion in higher dimensional Lorentz gases, thus our results depend on the following conjectures.

First, we assert that the leading order collision-free behaviour is determined only by maximal horizons, except where these are all incipient. This means in particular that incipient horizons, non-maximal horizons and overlaps between maximal horizons are all assumed to give subleading contributions.

Conjecture 1. Consider a periodic Lorentz gas with at least one non-incipient maximal horizon. Then we have

$$
\sum_{H \in \mathcal{H}} F_{H}(t) \sim F(t)
$$

as $t \rightarrow \infty$.

The initial sentence of the conjecture implies that $F(t)>0$ for all time as required by the right hand side of the equation. The definition of periodic Lorentz gas considered in this paper is given at the start of Sec. 4 .

Remark 1. This conjecture describes only the horizons and not reflections from the scatterers. It may not be necessary for the scatterers to be dispersing, as its content is purely geometrical, and not dynamical. The main obstruction to proving it is probably the effect of maximal incipient horizons (see the discussion preceding Conj. 2 below).

Remark 2. Where there are only incipient horizons, it is likely that $F(t)$ must decay to zero at finite time. We do not discuss this case here.

Now we are ready to state the first main result:

Theorem 1. Consider a periodic Lorentz gas satisfying Conjecture 1. Then the free flight function $F(t)$ satisfies

$$
F(t) \sim \sum_{H \in \mathcal{H}} \frac{S_{d_{H}-1} \int_{H_{x}^{\perp}} \int_{H_{x}^{\perp}} \Delta^{\mathrm{vis}}(\mathbf{x}, \mathbf{y}) d \mathbf{x} d \mathbf{y}}{S_{d-1} \mathcal{V}_{H}^{\perp}(1-\mathcal{P}) t^{d-d_{H}}}
$$

as $t \rightarrow \infty$.

Here, $\Delta^{\text {vis }}(\mathbf{x}, \mathbf{y})$ is the "visibility" counting function, giving the (non-negative integer) number of ways $\mathbf{x}$ and $\mathbf{y}$ can be connected by a straight line entirely in $H_{x}^{\perp}$; for the specific examples considered here, this is either zero or one. $S_{d}$ is defined in Tab. 1 .

Proof. Conjecture 1 asserts that only horizons in $\mathcal{H}$ need be considered to determine the asymptotic form of $F(t)$. Lemma 1 (below) shows that the sum over $\mathcal{H}$ is finite, and lemma 2 (below) gives the value of each term in the sum.

Sec. 6 gives the main application of this theorem, the expression (27) for $F(t)$ in the case of the cubic Lorentz gas with principal horizons.

If all maximal horizons are incipient, we cannot apply Conj. 1. However heuristic arguments and numerical simulations given in Sec. 7 suggest in the case of a principal incipient horizon, contributions from the infinite 
number of horizons of next lower dimension can be summed, giving a $t^{-2}$ decay of $F(t)$, as long as $d<6$. For $d \geq 6$ the sum of contributions diverges, and it appears that $F(t)$ has a decay intermediate between $t^{-2}$ and $t^{-1}$. Assuming a logarithm at the critical dimension $d=6$ we can rather tentatively suggest:

Conjecture 2. The free flight survival probability for a Lorentz gas with incipient (but no actual) principal horizon satisfies

$$
F(t) \asymp\left\{\begin{array}{ccc}
t^{-2} & & d<6 \\
t^{-2} \ln t & & d=6 \\
t^{-\alpha_{d}} \quad 1<\alpha_{d}<2, & d>6
\end{array}\right.
$$

Finally we turn to the (super-)diffusion coefficient. Here, a further conjecture is required to establish estimates on velocity autocorrelation functions. Consider a flight of length $t$ much larger than the lattice spacing; this has an angle from the horizon of order $t^{-1}$. The particle makes a grazing collision with a scatterer and reflects with a typical angle of order $t^{-1 / 2}$ so that the subsequent flight is typically of order $\sqrt{t}$ and further flights shorter still. Thus after any time proportional to $t$, we expect that many collisions have completely randomised the trajectories position relative to the scatterer and velocity direction, but not position in the full lattice. We state this as

Conjecture 3. Consider a periodic Lorentz gas with flow $\Phi^{t}$ and free flight function $F(t)>0$ for all $t>0$. Let $f, g: M \rightarrow \mathbb{R}$ denote zero-mean (with respect to $\mu$ ) Hölder functions. Then we have

$$
\int_{M \backslash M_{t}}(f)\left(g \circ \Phi^{t}\right) d \mu=o(F(t))
$$

as $t \rightarrow \infty$.

Note that the $\sqrt{t}$ motivating the above conjecture holds for the Lorentz gas, but not for other models such as extended stadium billiards. Also, a connection between free flights and correlations for the three dimensional cubic Lorentz gas was proposed as early as 1984 [19. We can now address the diffusion properties of the Lorentz gas:

Theorem 2. Consider a periodic Lorentz gas with non-incipient principal horizon. Assume Conjectures 1 and 3. Then the super-diffusion matrix is given by

$$
\mathcal{D}_{i j}=\frac{1}{1-\mathcal{P}} \frac{V_{d-1}}{S_{d-1}} \sum_{H \in \mathcal{H}} \frac{w_{H}^{2}\left(\delta_{i j}-n_{i}(H) n_{j}(H)\right)}{\mathcal{V}_{H}^{\perp}}
$$

where $\mathbf{n}(H)$ is either of the two unit vectors normal to all velocities in $H$.

Note that in contrast to two dimensions, it is preferable to describe horizons by their perpendicular rather than parallel directions, hence the projection operator appearing in this formula.

Combining both theorems and taking the trace of the diffusion matrix, we find the simple relation:

$$
d \mathcal{D}=\lim _{t \rightarrow \infty} t F(t)
$$

independent of the details of the principal horizons.

In the case that the principal horizons do not span the full space, the superdiffusion matrix is degenerate, and we expect that as in two dimensions, there will be normal diffusion in the direction(s) orthogonal to all these horizons if such motion is not completely blocked. Sec. 8 contains a numerical test of Conj. 3 and the proof of Thm. 2, with application to the discrete time diffusion coefficient given in Sec. 9.

For both the free flight function and the diffusion coefficient, the limit of small $r \rightarrow 0$ spherical scatterers leads to an increasing number of horizons. Taking a Mellin transform, both results can be formally related to the Riemann hypothesis $(\mathrm{RH})$, the celebrated conjecture that the complex zeros of the Riemann zeta function $\zeta(s)$ lie on the line with real part 1/2 [14]. The formal argument assumes that the contour integral can be closed avoiding the poles of $\zeta(s)^{-1}$ in the integrand in a convergent sequence of increasing semicircles. A complete proof of an equivalence with $\mathrm{RH}$ is not yet available even for the simpler related case of the open 
circular billiard of Ref. [6] work on the latter is ongoing. Connections between lattice sums and RH is of course not new; see for example [1. Here we make a connection between RH and transport in a definite physically motivated model; other physical systems with connections to RH are discussed in Ref. 41.

Further development of this subject could include verification and/or modification of the above conjectures on the theoretical side; the incipient case is particularly challenging due to the similarity with the example in Ref. 4, hence almost certainly exponential growth of complexity. The case of multiple rationally located scatterers per unit cell naturally leads to Dirichlet twisted Epstein zeta functions, which may be interesting from a number theoretical point of view (see the discussion at the end of Sec. 6). There are generalisations of the Lorentz gas which are almost periodic [18, 36] or have external fields [11, 12, 17.

On the applications side, periodic boundary conditions are commonly used in molecular dynamics simulations, so a natural extension would be to investigate the (possibly unphysical) effects of these boundary conditions in simulations of low density systems of many particles. Transport in periodic structures with long free flights occur naturally in crystalline materials, nanotubes and periodically fabricated devices such as antidot arrays and various classes of metamaterials. For these, it should be noted that the soft potentials may modify the dynamics of particles moving close to a horizon direction. As with periodic time perturbations, the effect could be either more or less stable than free motion in the Lorentz gas depending on the details of the potential and the speed of the particle, however, as here, enumeration of horizons remains important for understanding transport properties.

The remaining sections are structured as follows: Sec. 4 motivates and gives the necessary definitions related to the horizons, Sec. 5 develops the proof of Thm. 1. proving two lemmas and discussing Conj. 1 . Sec. 6 then applies this theorem to principal horizons, giving $F(t) \sim C / t$ with the constant determined exactly. Numerical methods are then discussed, and used to confirm these results. In the limit of small scatterers, connection is made with the Riemann Hypothesis. Sec. 7 then considers incipient horizons, where heuristic arguments and numerical simulations suggest Conj. 2 . Sec. 8 then turns to the diffusion problem, proving Thm. 2 conditional on Conjs. 1 and 3 , again for principal horizons. In this case the numerical results are inconclusive due to the logarithm being dominated by a constant at accessible numerical timescales. Finally, Sec. 9 treats the diffusion problem in discrete time.

\section{Definitions}

The phase space of the full periodic Lorentz gas is $\tilde{M}=\tilde{M}_{x} \times M_{v}$ where $\tilde{M}_{x} \subset \mathbb{R}^{d}$ denotes the configuration space external to the scatterers and $M_{v}=\mathbb{S}^{d-1}$ is the set of velocities of unit magnitude. The same quantities without tildes are associated with the compact phase space of the dynamics considered modulo the lattice, so $M=M_{x} \times M_{v}$ with $M_{x}=\mathbb{R}^{d} / \mathcal{L} \backslash \Omega$. There are natural projections $\pi_{x}: M \rightarrow M_{x}$ and $\pi_{v}: M \rightarrow M_{v}$. Here, $\mathcal{L}$ is the lattice of periodicity of the Lorentz gas, ie a discrete subgroup of $\mathbb{R}^{d}$ with finite covolume $\mathcal{V}$, and in the main example given by $\mathbb{Z}^{d}$. Also, $\Omega \subset \mathbb{R}^{d} / \mathcal{L}$ denotes the union of the scatterers. The scatterers (connected components of $\Omega$ ) are assumed to be finite in number. The curvature of their boundaries has a positive lower bound, and the boundaries themselves are $C^{3}$ smooth. To allow for touching or overlapping spheres (for example), the scatterer boundaries may be non-smooth on a finite number of submanifolds of dimension at most $d-2$; we require however that each scatterer be the union of a finite number of convex sets in this case.

The uniform probability measure on $M$ is $\mu=m /\left[\mathcal{V} S_{d-1}(1-\mathcal{P})\right]$ where $m$ is the usual Lebesgue measure for $\mathbb{R}^{d} \times \mathbb{S}^{d-1}, S_{d-1}$ is the Lebesgue measure of the sphere $\mathbb{S}^{d-1}$ (see Tab. 1) and $\mathcal{P}$ is the packing fraction of the obstacles, equal to $m_{x}(\Omega) / \mathcal{V}$ where $m_{x}$ is Lebesgue measure on $\mathbb{R}^{d}$.

The usual billiard flow is denoted $\Phi^{t}: M \rightarrow M$. Thus we have the free flight formula $\Phi^{t}(\mathbf{x}, \mathbf{v})=$ $(\mathbf{x}+\mathbf{v} t \bmod \mathcal{L}, \mathbf{v})$ in the absence of a collision. The time to the next collision is denoted $T(\mathbf{x}, \mathbf{v})=\inf \{t>$ $\left.0: \pi_{x} \circ \Phi^{t}(\mathbf{x}, \mathbf{v}) \in \partial \Omega\right\}$. If there is no future collision, we allow $T(\mathbf{x}, \mathbf{v})$ to be infinite. The set surviving for time $t$ without a collision is $M_{t}=\{(\mathbf{x}, \mathbf{v}) \in M: T(\mathbf{x}, \mathbf{v}) \geq t\}$, in terms of which $F(t)=\mu\left(M_{t}\right)$. One of our main aims is to characterise the large $t$ asymptotics of this function.

The flow $\Phi^{t}$ is discontinuous at a collision, using the usual mirror reflection law, but we do not need the explicit formula here. Nor do we need to specify conventions for the flow at the exact moment of collision or for orbits that are tangent to the boundary or reach a non-smooth point on it; these sets are of zero measure. 
In three or more dimensions, collections of orbits with large or infinite $T$ are of different types. We now formalise the terms introduced in the previous section. We want to characterise subsets of $M_{\infty}$, ie collections of points such that $T=\infty$.

The free dynamics is uniform motion on a torus. Thus a trajectory starting from a point in $M$ must either be dense (hence have finite $T$ ) or move parallel to a linear subspace spanned by vectors in $\mathcal{L}$. Thus for each $\mathbf{x} \in \pi_{x} M_{\infty}$ there are one or more (possibly infinitely many) linear subspaces of directions in which there are no collisions. Let us choose one, $V_{H}$ which is not contained in another such subspace. Then, we can define

$$
\begin{aligned}
& H_{v}=V_{H} \cap \mathbb{S}^{d-1} \\
& \hat{H}_{x}=\left\{\mathbf{x} \in M_{x}:\left(\mathbf{x}, H_{v}\right) \in M_{\infty}\right\}
\end{aligned}
$$

The equation defining free motion ensures that $\hat{H}_{x}$ is invariant under translations in $V_{H}$. Thus we can decompose it as

$$
\hat{H}_{x}=\hat{H}_{x}^{\perp} \oplus V_{H} / \mathcal{L}_{H}
$$

where $\mathcal{L}_{H}=\mathcal{L} \cap V_{H}$ is a sublattice of the original lattice $\mathcal{L}$. Initial conditions contained in $\hat{H}_{x}$ and having velocities close to vectors in $H_{v}$ will survive for a long time before colliding, as long as they remain in a connected component of $\hat{H}_{x}$. This motivates the following definition of a horizon:

Definition 1. A Horizon is a set $H \subset M_{\infty}$ which satisfies

(a) $H$ is a direct product of sets $H_{x} \subset M_{x}$ and $H_{v} \subset M_{v}$,

(b) $H$ is inextensible, ie not a proper subset of another horizon,

(c) $H_{x}$ is connected.

The above discussion implies that all horizons are of the form given in Eqs. 2022 above, with the additional condition that $H_{x}$ be a connected component of $\hat{H}_{x}$, that is,

$$
\begin{aligned}
H & =H_{x} \times H_{v} \\
H_{x} & =H_{x}^{\perp} \oplus V_{H} / \mathcal{L}_{H} \\
H_{v} & =V_{H} \cap \mathbb{S}^{d-1}
\end{aligned}
$$

The dimension of the horizon $d_{H} \in\{1 \ldots d-1\}$ is defined to be the dimension of $V_{H}$, which is also the dimension of $\mathcal{L}_{H}$. When $d_{H}=1, H_{v}$ consists of two opposite points, so $H$ itself is not connected, even though $H_{x}$ is connected. When $d_{H}>1, H$ is also connected.

An example where $\hat{H}_{x}$ may have more than one connected component is that of a square lattice, ie $\mathcal{L}=\mathbb{Z}^{2}$, with a circular scatterer of radius $r_{1}=0.2$ at the origin and an additional scatterer of radius $r_{2}=0.1$ at $(1 / 2,1 / 2)$. Particles moving horizontally will not hit any scatterer if their $y$ coordinate lies in either of the intervals $(0.2,0.4),(0.6,0.8)$. Thus there are two horizons with the same $V_{H}$ in this case.

We denote the $d-d_{H}$ dimensional covolume of $\mathcal{L}_{H}$ in $V_{H}$ by $\mathcal{V}_{H}$. The space of dimension $d-d_{H}$ perpendicular to $V_{H}$ is denoted $V_{H}^{\perp}$. The original lattice induces a lattice $\mathcal{L}_{H}^{\perp} \subset V_{H}^{\perp}$, given by elements of $\mathcal{L} / \mathcal{L}_{H}$ projected onto $V_{H}^{\perp}$ along $V_{H}$. This has covolume $\mathcal{V}_{H}^{\perp}=\mathcal{V} / \mathcal{V}_{H}$ since a unit cell of the original lattice can be formed by the product of two unit cells of lower dimension in orthogonal subspaces. In the original space $\tilde{M}$ each horizon $H$ corresponds to a periodic collection of connected components; lattice translations in $\mathcal{L}_{H}$ preserve each component, while lattice translations in $\mathcal{L} \backslash \mathcal{L}_{\mathcal{H}}$ map one component to another.

When $d=2$ we can have only horizons with dimension $d_{H}=1$, corresponding to the channels in previous literature (see Sec. 2). When $d=3$ we can have a "planar horizon", $d_{H}=2$, in which $H_{v}$ is a great circle in $\mathbb{S}^{2}$, a "cylindrical horizon", $d_{H}=1$, in which $H_{v}$ consist of two symmetrically placed points; this terminology comes from [37. More than one type of horizon may exist in the same billiard. For example if the scatterers do not cross the boundaries of the unit cell in three dimensions, there are planar horizons along the boundaries; there may also be a horizon of cylindrical type in the interior. Finite horizon, in which no horizons exist, are also possible in any dimension $d$, and becomes increasingly probable for random 
arrangements of scatterers of fixed density in a unit cell of increasing size. Nevertheless no explicit example of a finite horizon periodic Lorentz gas in $d>2$ has yet been shown.

Recall the discussion of the free flight function, $F(t)$ above. We can similarly define the probability of remaining for at least a given time in the spatial projection of a horizon, $F_{H}(t)$; this definition has already been given in Eq. (14). It is useful to make some definitions related to the dimension:

Definition 2. Maximal horizon: A horizon of dimension greater than or equal to all other horizons of the given billiard.

Definition 3. Principal horizon: A horizon $H$ of the highest possible dimension, that is, $d_{H}=d-1$.

Clearly a principal horizon is also maximal. Anomalous diffusion properties arising from a $1 / t$ decay of velocity autocorrelation functions are expected only for principal horizons; see Ref. [33, 37] and below.

We now make an important observation: For dimensions $d>2$ we have a qualitatively new type of horizon, corresponding to the border between horizons of one dimension and the next.

Definition 4. Incipient horizon: A horizon for which $H_{x}^{\perp}$ has zero $d-d_{H}$ dimensional Lebesgue measure. An incipient maximal or incipient principal horizon will denote a horizon that is incipient and also maximal or principal, respectively.

When $d=2$, an incipient horizon is an orbit tangent to scatterers on both sides, hence isolated and having no effect on the dynamics; this is a rather trivial form of horizon. In dimensions $d>2$, a incipient horizon occurs when $H_{x}^{\perp}$ for a horizon of dimension $d_{H}>1$ shrinks to a zero measure set, so that $F_{H}(t)=0$. This is easiest to visualise in the three dimensional cubic Lorentz gas, when the planar horizons shrink to zero width touching scatterers on both sides as the radius approaches $1 / 2$ from below; see Fig. 2 .

Within an incipient horizon $H$, there is a lattice of points $\mathcal{L}_{i n c}$ in $H_{x}$ where the scatterers touch the horizon. The set of velocities within $H_{v}$ that do not come arbitrarily close to the scatterers consists of those contained in horizons for a Lorentz gas of dimension $d_{H}$ with infinitesimal scatterers placed at points of $\mathcal{L}_{\text {inc }}$. Each of these velocities corresponds to a horizon of dimension $\leq d_{H}-1$ in $H$ and also in the original space $M$. However, unlike in the Lorentz gas with finite sized scatterers, the number of horizons of the highest dimension $d_{H}-1$ is now countably infinite, parallel to all possible $d_{H}-1$ dimensional subspaces spanned by vectors in $\mathcal{L}_{\text {inc }}$. These become finite in number as soon as the radius of the scatterers is increased and the incipient horizon destroyed. Thus an incipient horizon of dimension $d_{H}>1$ is in general associated with an infinite number of horizons of dimension $d_{H}-1$. This leads to the interesting effects discussed in Conj. 2 above and Sec. 7 below.

We also remark that incipient horizons contain orbits tangent to infinitely many scatterers from more than one direction, like the very recent example of exponential growth of complexity found in finite horizon multidimensional billiards 4 . Here too, the singularity structure in the vicinity of such orbits is extremely complicated. One final definition:

Definition 5. $\mathcal{H}$ is the set of non-incipient maximal horizons.

\section{Two lemmas}

We now consider the first two propositions in section 2 of Bleher [5] in the more general case of arbitrary dimension, which are necessary for the proof of Thm. 1. The first proposition can be naturally generalised, and is given as the first lemma here. The second proposition is not valid in $d>2$, and must be replaced by Conj. 1 above. The contribution from each horizon, also needed for Thm. 1 , forms the content of the second lemma.

We generalise Bleher's [5] Prop. 2.1:

Lemma 1. For any periodic Lorentz gas with infinite horizon there are finitely many maximal (possibly incipient) horizons. 
Proof. Assume there are an infinite number of maximal horizons, each with its velocity subspace $V_{H}$. The set of $V_{H}$ is either finite or infinite. If it is finite, at least one $V_{H}$ corresponds to infinitely many $H$, so that $\hat{H}_{x}$ has infinitely many connected components. But this is not possible since the scatterers are comprised of only a finite number of convex pieces. Thus we conclude there are also an infinite number of $V_{H}$, leading to unbounded $d_{H}$-dimensional covolumes $\mathcal{V}_{H}$. The horizons thus fill at least one space of dimension greater than $d_{H}$ more and more densely, which is only possible if the intersection of the interior of the scatterers with this space is empty. Thus the space forms its own (possibly incipient) horizon with a dimension greater than $d_{H}$, violating the assumption that the original horizons are maximal.

This lemma ensures that the sum over maximal horizons appearing in Thm. 1 is finite.

Bleher's [5] Prop. 2.2 asserts that in any sufficiently long free path (greater than a fixed constant $R_{0}$ ), all but extreme parts of bounded (less than another constant $R_{1}$ ) total length lie in the space projection $H_{x}$ of a horizon $H$, or in the terminology of that paper, a corridor. For $d>2$ this is not true, since there is in general a complicated set of horizons of differing dimension connected in a nontrivial manner.

All we can hope for is conjecture 1 given in the introduction, which we now discuss in more detail. The sum over $H \in \mathcal{H}$ ignores lower dimensional horizons, some of which are connected to the maximal horizons. It also double counts overlaps of the maximal horizons. For example, in the three dimensional Lorentz gas, with a cubic arrangement of spherical scatterers of radius $r<1 / 2$ (considered in more detail later), there are planar horizons parallel to the $(x, y),(x, z)$ and $(y, z)$ planes, for example the set $\left\{(x, y, z) \in \mathbb{R}^{3}: r<\right.$ $z<1-r\}$. Any two of these intersect in cylindrical sets parallel to one of the coordinate axes. There are also lower dimensional (ie cylindrical) horizons that are not part of the planar horizons, for example the set $\left\{(x, y, z) \in \mathbb{R}^{3}: 0<x, y<r, x^{2}+y^{2}>r^{2}\right\}$. Conjecture 1 asserts that these lower dimensional effects do not affect the leading order free flight function $F(t)$ if there is a non-incipient maximal horizon.

The exclusion of purely incipient maximal horizons is necessary, as in this case $F_{H}(t)=0$. For $d_{H}>1$ this is associated with an infinite number of horizons of lower dimension, whose contributions (including intersections) would need to be carefully enumerated to calculate $F(t)$; we give some heuristic arguments and numerical simulations for the case of incipient principal horizons in Sec. 7 .

We now come to the most important result of this section:

Lemma 2. For a non-incipient horizon $H$, we have as $t \rightarrow \infty$

$$
F_{H}(t) \sim \frac{S_{d_{H}-1} \int_{H_{x}^{\perp}} \int_{H_{x}^{\perp}} \Delta^{\mathrm{vis}}(\mathbf{x}, \mathbf{y}) d \mathbf{x} d \mathbf{y}}{S_{d-1} \mathcal{V}_{H}^{\perp}(1-\mathcal{P}) t^{d-d_{H}}}
$$

Proof. We split $\mathbf{v}$ into components in $V_{H}^{\perp}$ and $V_{H}$ subspaces: $\mathbf{v}=\mathbf{v}^{\perp}+\mathbf{v}^{\|}$, and similarly for $\mathbf{x}$. The trajectories not reaching the boundary of the horizon before time $t$ are given by $\mathbf{v}^{\perp} \in\left(\tilde{H}_{x}^{\text {vis }}(\mathbf{x})-\mathbf{x}\right) / t$ to leading order in $t$. Here, $\tilde{H}_{x}^{\text {vis }}(\mathbf{x})$ is the set of points "visible" from $\mathbf{x}$ in $H_{x}^{\perp}$, ie the set connected by a straight line that does not leave $H_{x}^{\perp}$. The tilde indicates that this set is defined on the full $V_{H}^{\perp}$, not modulo the lattice, ie more than one lattice translate of a point may be visible to a given $\mathbf{x} \in H_{x}^{\perp}$.

The definition of $F_{H}(t)$ is given in Eq. (14). Now $\mu$ is just given by $m /\left[\mathcal{V} S_{d-1}(1-\mathcal{P})\right]$ where $\mathcal{P}$ is the packing fraction of scatterers and $m$ is Lebesgue measure. We integrate over all $\mathbf{x}^{\|}$which is the covolume of $\mathcal{L}_{H}$ in $V_{H}, \mathcal{V}_{H}$. The measure over $\mathbf{x}^{\perp}$ and $\mathbf{v}^{\perp}$, multiplied by $t^{d-d_{H}}$, is given by the integral

$$
\int_{H_{x}^{\perp}}\left|\tilde{H}_{x}^{\text {vis }}(\mathbf{x})\right| d \mathbf{x}
$$

which can be written more symmetrically as

$$
\int_{H_{x}^{\perp}} \int_{H_{x}^{\perp}} \Delta^{\mathrm{vis}}(\mathbf{x}, \mathbf{y}) d \mathbf{x} d \mathbf{y}
$$

Since $\mathbf{v}^{\perp}$ is of order $t^{-1}, \mathbf{v}^{\|}$remains of unit magnitude to leading order, and its measure is simply $S_{d_{H}-1}$. Taking account of the normalisation factors and the relation $\mathcal{V}=\mathcal{V}_{H} \mathcal{V}_{H}^{\perp}$ we get the result stated in the lemma. 


\section{Principal horizons and the free flight function}

In the case of a principal horizon, we note that $H_{x}^{\perp}$ is one dimensional, so that the double integral in Lemma 2 is simply $\left|H_{x}^{\perp}\right|^{2}$. Otherwise $H_{x}^{\perp}$ is typically non-convex and the integral is not easy to evaluate (an example is provided in the next section).

Possible principal horizons can be enumerated using the dual lattice $\mathcal{L}^{*}=\left\{\mathbf{u} \in \mathbb{R}^{d}: \mathbf{u} \cdot \mathbf{v} \in \mathbb{Z}^{d}, \quad \forall \mathbf{v} \in \mathcal{L}\right\}$ where $\mathbf{u} \cdot \mathbf{v}$ denotes the usual inner product in $\mathbb{R}^{d}$. In particular, for each lattice $\mathcal{L}_{H}$ of dimension $d-1$, either of the two vectors perpendicular to $\mathcal{V}_{H}$ and of length $\left(\mathcal{V}_{H}^{\perp}\right)^{-1}$ lie in $\mathcal{L}^{*}$. It is not hard to see that there is a 1:1 correspondence between codimension- 1 lattices and primitive elements of $\mathcal{L}^{*}$ modulo inversion, where primitive indicates that it is not a multiple of an element of $\mathcal{L}^{*}$ by an integer other than \pm 1 , and inversion is multiplication by -1 . Note that the zero vector is not primitive. We denote such a vector by $\mathbf{l}=\mathbf{n}_{H} / \mathcal{V}_{H}^{\perp}$ with magnitude $L=|\mathbf{l}|=\left(\mathcal{V}_{H}^{\perp}\right)^{-1}$ so that $\mathbf{n}_{H}$ is a unit vector perpendicular to $V_{H}$. The subscript $H$ is suppressed for the vectors $\mathbf{l}$ and their magnitudes $L$. For each principal horizon there is an ambiguity in the sign of $\mathbf{l}$ and $\mathbf{n}_{H}$ that does not affect any of the results. When summing over all primitive lattice vectors, the set denoted $\hat{\mathcal{L}}^{*}$, we need to divide by 2 to take account of this inversion symmetry.

Given a vector $\mathbf{l} \in \hat{\mathcal{L}}^{*}$, a principal horizon will exist if the perpendicular projection of the scatterers onto the line parallel to $\mathbf{l}$ does not cover the whole of that line. If there is one spherical scatterer of radius $r$ per unit cell, this corresponds to the condition that the width of the horizon $w_{H}=\mathcal{V}_{H}^{\perp}-2 r=L^{-1}-2 r$ is non-negative. Thus for a given $r$, there are a finite number of principal horizons corresponding to vectors in $\hat{\mathcal{L}}^{*}$ modulo inversion with lengths less than $(2 r)^{-1}$; this number grows without bound as $r$ approaches zero. For a finite number of arbitrary convex scatterers, this argument gives an upper bound if $2 r$ is interpreted as the smallest width of any of the scatterers.

Returning to the case of a single spherical scatterer of radius $r$ per unit cell, we have $\mathcal{P}=V_{d} r^{d}$ (see Tab. 1). Using Thm. 1, we find that

$$
F(t) \sim \frac{G_{d}}{t\left(1-V_{d} r^{d}\right)} \sum_{\mathbf{l} \in \hat{\mathcal{L}}^{*}} L u_{2}\left(L^{-1}-2 r\right)
$$

where $u_{2}(x)=x^{2} u(x), u(x)$ the unit step function, equal to 1 for positive argument and zero otherwise, and $L=|\mathbf{1}|$ as above. The geometrical factor $G_{d}=S_{d-2} /\left(2 S_{d-1}\right)$ is given in Tab. 1 , where the 2 comes from the inversion symmetry.

Numerical simulations were performed using the author's $\mathrm{C}++$ code, which is designed to simulate arbitrary piecewise quadric billiards in arbitrary dimensions. The flight time is thus given the solution of a quadratic equation; simplifications for the cases where the matrix defining the quadratic form is zero or the unit matrix are used for greater efficiency. The main numerical difficulty with billiards is to ensure that the particle remains on the correct side of the boundary, while retaining as much accuracy as possible for almost tangential collisions. These can be addressed by disallowing very rapid multiple collisions within a very small perpendicular distance of the boundary. Tests were made comparing 16 and 19 digit precision calculations, and by an explicit investigation of almost tangential collisions.

For the calculations presented here, the spherical scatterer is contained in a cubic cell. On reaching the boundary, the particle is transferred to the opposite side, updating the local position while keeping track of the overall displacement. The main obstacle to simulations in dimensions above $d=10$ considered here is that the volume fraction of balls decreases rapidly with dimension for the cubic lattice. Other lattices (for example the close packed $E_{8}$ lattice) may be considered in the future, however a higher volume fraction comes at the cost of a more complicated unit cell. It is difficult to get an intuitive grasp of the structure of horizons of non-cubic lattices above three dimensions; one approach would be to numerically infer them from $F(t)$ via Thm. 1 .

Numerical simulations of $F(t)$ were performed for the case of a cubic lattice with $r=0.4$ where there is only a single contribution in the above sum, for $2 \leq d \leq 10$ in Fig. 3 . Agreement is good for low dimensions, while for higher dimensions it is plausible but limited by finite statistics together with longer times required for convergence to the final $C / t$ form. In the case of $r=0.6$ the scatterers are overlapping, and the maximal horizons have dimension $d-2$. Here we expect $C / t^{2}$ behaviour for $d \geq 3$ but do not have an explicit analytic formula; see Fig. 4 which shows good qualitative agreement for small dimensions, and slow convergence for 


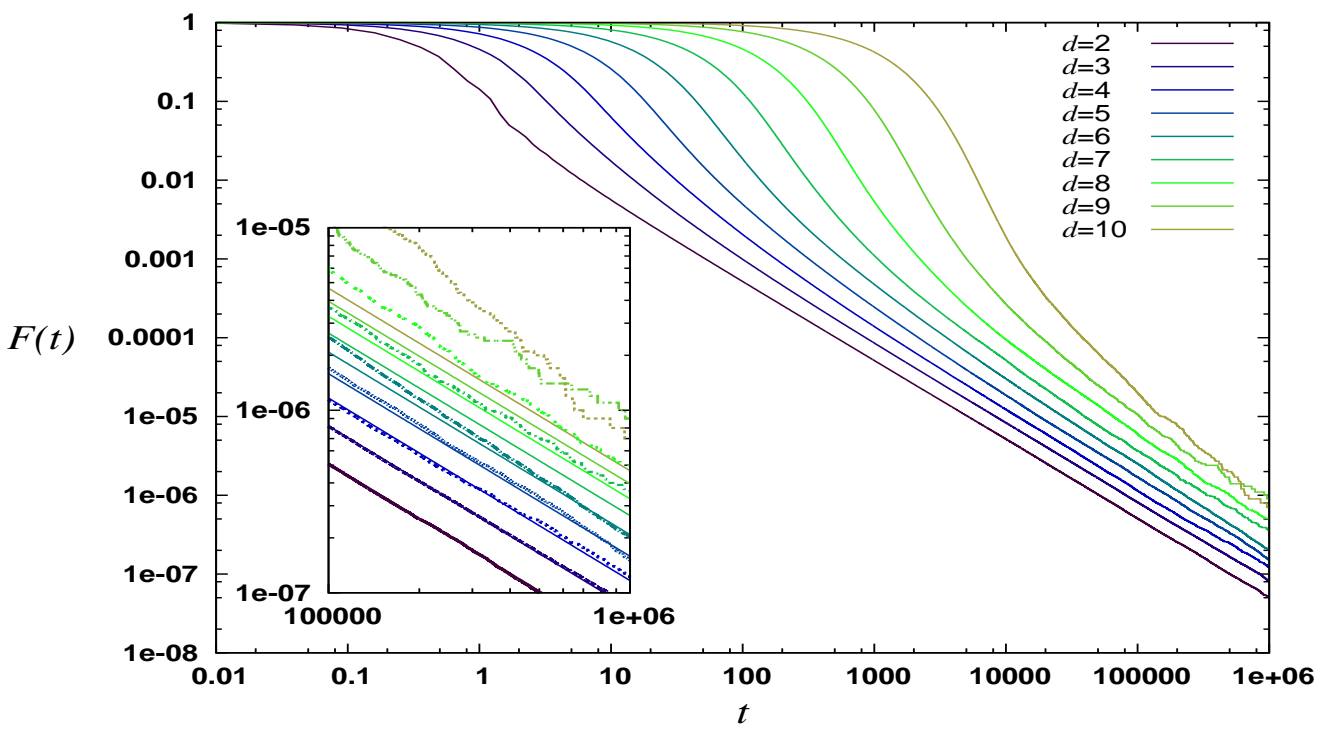

Figure 3: The free flight function for dimensions $2 \leq d \leq 10$ for $r=0.4$ showing $C / t$ behaviour. The inset shows comparison with the predictions of Eq. 27); here the numerical results are dotted and the predictions the solid straight lines.

higher dimensions. The intermediate case of $r=0.5$ (incipient principal horizon) is considered in section 7 below.

We now seek the small $r$ asymptotics of the above expression. First we remove the primitivity condition using Möbius inversion,

$$
F(t)=\frac{G_{d}}{t\left(1-V_{d} r^{d}\right)} \sum_{C=1}^{\infty} \sum_{\mathbf{l} \in \mathcal{L}^{*}}^{\prime} \mu(C) C L u_{2}\left((C L)^{-1}-2 r\right)
$$

where the prime indicates that the sum excludes the zero vector. We take out the geometric factor and use a Mellin transform on the rest to extract the small $r$ behaviour

$$
F(t)=\frac{G_{d}}{t\left(1-V_{d} r^{d}\right)} \frac{1}{2 \pi i} \int_{c-i \infty}^{c+i \infty} \frac{4 r^{-s} E\left((s+1) / 2 ; \mathcal{L}^{*}\right) d s}{2^{s+1} s(s+1)(s+2) \zeta(s+1)}
$$

where $E\left(s^{\prime} ; \mathcal{L}^{*}\right)$ is the Epstein zeta function for the dual lattice,

$$
E\left(s^{\prime} ; \mathcal{L}^{*}\right)=\sum_{1 \in \mathcal{L}^{*}}^{\prime} L^{-2 s^{\prime}}
$$

The purpose of the Mellin transform is to express the contour integral as a sum of residues by Cauchy's theorem, closing the contour to the left. This sum over poles directly gives an expansion in powers of $r$ (also logarithms if the poles are not single). It naturally depends on being able to show that there exists a sequence of roughly semicircular contours for negative real part of $s$ and avoiding poles of the integrand, with integral converging to zero; we are unable to prove this here.

The integrand includes the Riemann zeta function $\zeta(s)$ in the denominator, so the powers of $r$ appearing in the residue sum depend on the locations of the zeros of $\zeta(s)$. The celebrated Riemann Hypothesis 14 ] 


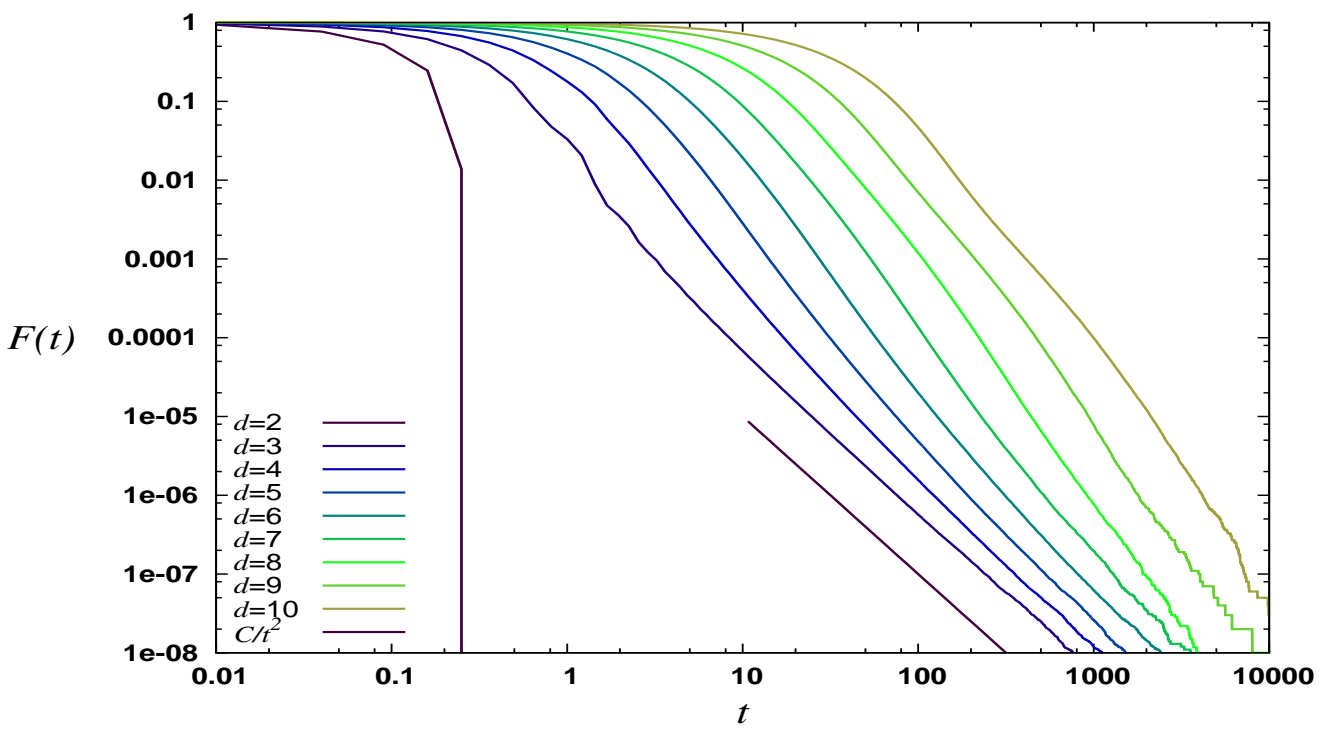

Figure 4: The free flight function for dimensions $2 \leq d \leq 10$ for $r=0.6$ (overlapping) scatterers, showing a collision at bounded time for $d=2$ and approach to $C / t^{2}$ for $d>2$; the rate of convergence with $t$ is slow for the higher dimensions considered.

asserts that the complex zeros lie on the line with real part of $s$ equal to $1 / 2$; the zeros on the real axis are all known.

We also need some analytic properties of the Epstein zeta function in the numerator; see 38 . The function $E\left(z, \mathcal{L}^{*}\right)$ for arbitrary lattice of full dimension $\mathcal{L}^{*}$ has an analytic continuation in the whole complex plane except for a single pole at $z=d / 2$ with residue $S_{d-1} / 2$, a special value $E\left(0 ; \mathcal{L}^{*}\right)=-1$ and zeros at all negative integers. The following statements 43 show how it generalises the Riemann zeta function $\zeta(s)$ : Considering the (self-dual) cubic lattice $\mathcal{L}=\mathcal{L}^{*}=\mathbb{Z}^{d}$, we have $E\left(s^{\prime} ; \mathbb{Z}\right)=2 \zeta\left(2 s^{\prime}\right)$ and $E\left(s^{\prime} ; \mathbb{Z}^{2}\right)=4 \zeta\left(s^{\prime}\right) \beta\left(s^{\prime}\right)$ where $\beta$ is the Dirichlet $\beta$-function, that is, the nontrivial Dirichlet $L$-function modulo 4 . In four dimensions we have $E\left(s^{\prime} ; \mathbb{Z}^{4}\right)=8\left(1-2^{2-2 s^{\prime}}\right) \zeta\left(s^{\prime}\right) \zeta\left(s^{\prime}-1\right)$, which is already enough to show that the Epstein zeta functions do not all satisfy a Riemann hypothesis with all complex zeros on a single vertical line in the complex plane; note that Ref. 43 has $2^{2-s^{\prime}}$ (a misprint). There is no factorisation for the physically interesting case of three dimensions, as for example there are lattice vectors with lengths $\sqrt{3}$ and $\sqrt{5}$ but not the product $\sqrt{15}$.

The above integral has poles at $s=d-1,-1,-2$ and at complex values with real part $-1 / 2$ due to the $\zeta(s+1)$ in the denominator (also elsewhere if the Riemann Hypothesis is false). Assuming that the contour can be continued to the left, avoiding poles of the Riemann zeta function, we find for the $d$-dimensional cubic lattice,

$$
t F(t)=\frac{\pi^{(d-1) / 2}}{2^{d} d \Gamma((d+3) / 2) \zeta(d) r^{d-1}}+O\left(r^{1 / 2-\delta}\right)
$$

where the leading coefficient is the same as found for the Boltzmann-Grad limit in [31, and the next term is valid for all $\delta>0$ if the Riemann Hypothesis is true. Numerically this term is small in magnitude for $r$ not too small; the next term

$$
\left[\frac{S_{d-2} V_{d}}{2^{d-1}\left(d^{3}-d\right) \zeta(d)}-8 G_{d}\right] r
$$

is quite visible; see Fig. 5 . This argument with the contour is similar to that of the survival probability of the circular billiard, Ref. [6], and as in that case suggests an equivalence of Eq. 31 to the Riemann Hypothesis, 


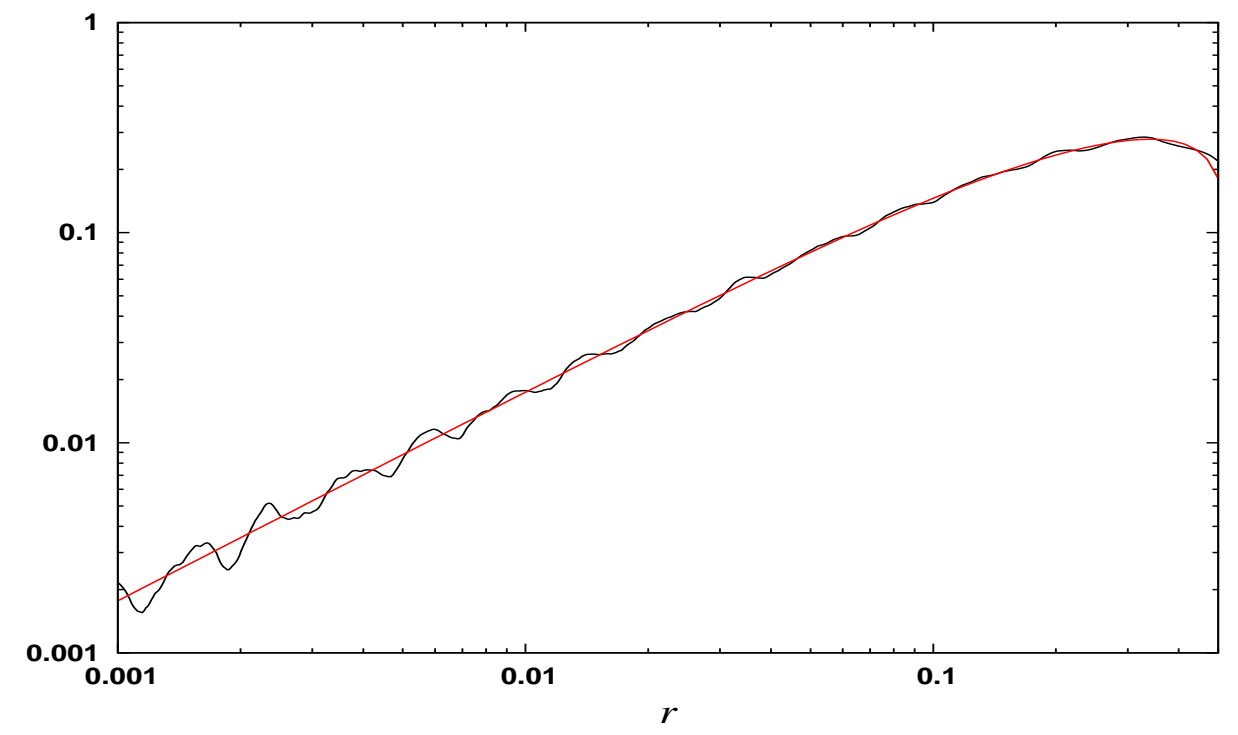

Figure 5: The sum in Eq.27) minus the leading term in Eq. (31), compared with the series obtained by summing the residues of poles on the real axis in Eq. $(29)$, the smooth curve. The smooth terms give a good approximation for the range of $r$ considered; for smaller $r$ the complex poles coming from the zeta function dominate.

requiring a further careful argument.

In the case of two or more scatterers per unit cell with rationally located centres and common denominator $q$, the same calculation can be performed. The Mellin transform then gives an expression of the form

$$
t F(t)=\sum_{\chi} f_{\chi}(s) \sum_{\mathbf{l} \in \mathcal{L}^{*}}^{\prime} \frac{\chi(\mathbf{l} \cdot \mathbf{m})}{L^{s+1}} \sum_{C=1}^{\infty} \frac{\mu(C) \chi(C)}{C^{s+1}}
$$

where $\chi$ is a Dirichlet character mod $q, f_{\chi}(s)$ and $\mathbf{m}$ are explicitly known, the sum over $C$ can be expressed in terms of known Dirichlet $L$-functions, relating transport in this Lorentz gas to the generalised Riemann Hypothesis. The sum over $\mathbf{l}$ gives Dirichlet-twisted Epstein zeta-functions. The latter may be difficult to characterise in $d=3$ due to the lack of a factorisation of the untwisted Epstein zeta function.

\section{Incipient horizons and the critical dimension}

The results of the last section do not apply directly to the case of incipient principal horizons, as their width is zero, and they are associated with an infinite number of horizons of the next lower dimension. There is however at least one useful piece of information to be gained, which we now describe.

Lemma 2 shows that the contribution to $F(t)$ from a horizon of dimension $d-2$ is of order $t^{-2}$. A finite number of these contributions are thus of the same order, however a more careful calculation is required for the present case, where there are an infinite number of contributions.

We consider a single example, but one which is likely to have more general implications. Consider the Lorentz gas with a cubic lattice of arbitrary dimension $d>2$ and $r=1 / 2$. The scatterers just touch, leading to incipient horizons in the coordinate planes and no other principal horizons. In one of these planes, for example at $x_{d}=1 / 2$ for the $d$-th coordinate, the cross-section of the scatterers are reduced to the points of 


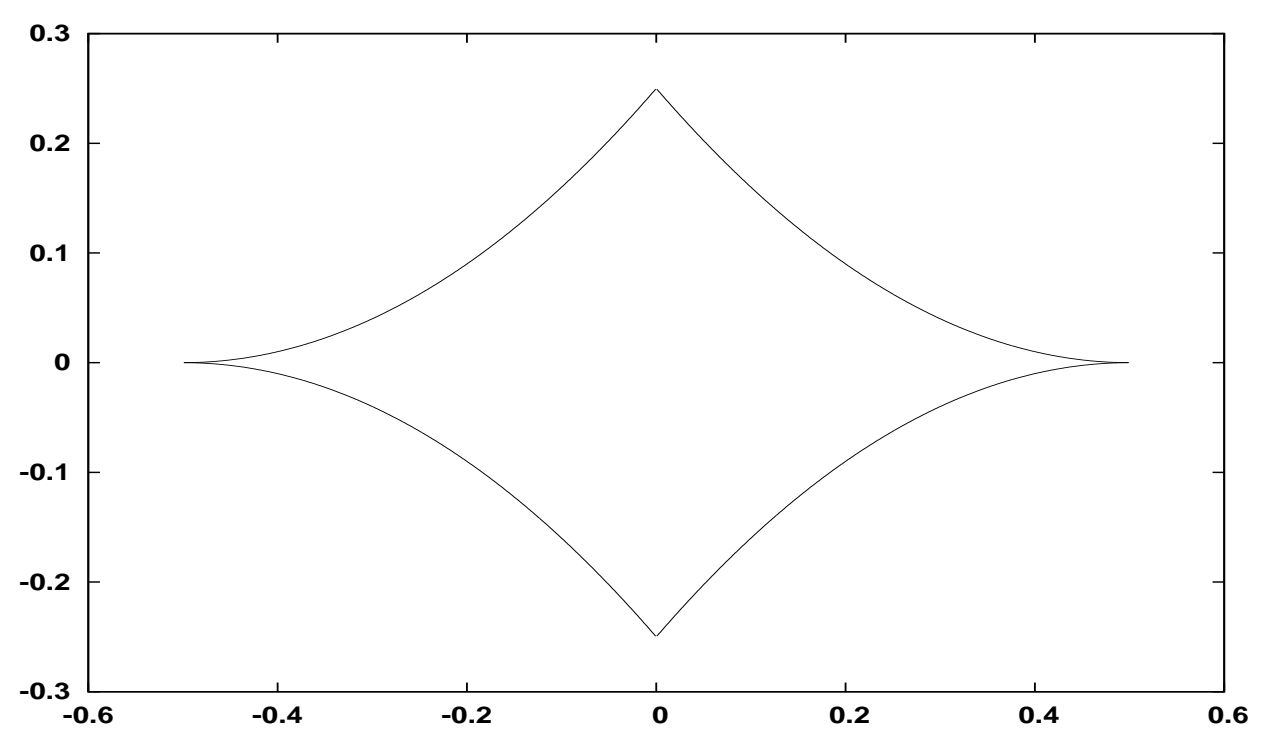

Figure 6: The region defined by Eq. 33 .

$\mathbb{Z}^{d-1}$ in the other $d-1$ coordinates, equivalent to the problem in the previous section, with dimension $d-1$ and $r=0$. Thus there are horizons of dimension $d-2$ corresponding to all non-zero lattice vectors $\mathbf{l} \in \hat{\mathbb{Z}}^{d-1}$ modulo inversion. For any given lattice vector, there will also be a finite extent in the $d$-th coordinate direction, which we now quantify.

The space $H_{x}^{\perp}$ is two dimensional, in the plane spanned by $\mathbf{l}$ and $\mathbf{e}_{d}$, the unit vector in the $d$-th coordinate. Projected orthogonally into this plane, the lattice has basis vectors $1 / L^{2}$ (hence of magnitude $L^{-1}$, where $L=|\mathbf{1}|$, consistent with previous notation) and $\mathbf{e}_{d}$. This means $H_{x}^{\perp}$ consists of the area confined between four circles of radius $r=1 / 2$ and centred at $(0,0),\left(L^{-1}, 0\right),(0,1),\left(L^{-1}, 1\right)$ with respect to the orthonormal basis $\left\{\mathbf{l} / L, \mathbf{e}_{d}\right\}$; we will denote coordinates with respect to this basis as $(x, y)$. Pairs of scatterers separated by $L^{-1}$ overlap (except when $L=1$ ) and pairs displaced by 1 just touch. The region not within radius $r=1 / 2$ of the centre of any scatterer is contained in the rectangle defined by $0<x<L^{-1}$ and $\frac{1}{2} \sqrt{1-L^{-2}}<y<$ $1-\frac{1}{2} \sqrt{1-L^{-2}}$. In the limit as $L \rightarrow \infty$ the height in the $y$ direction is $L^{-2} / 2$ to leading order. In this limit, we scale in both directions, $x-L^{-1} / 2=L^{-1} \xi$ and $y-1 / 2=L^{-2} \eta$ and keep only the highest order nontrivial terms in the equation, and find that $H_{x}^{\perp}$ is defined by a limiting shape bounded by four parabolas,

$$
|\eta|<(|\xi|-1 / 2)^{2}, \quad|\xi|<1 / 2
$$

This region is plotted in Fig. 6. There is thus a limiting nonzero probability for two points in the region to be mutually "visible", ie for which the line joining the points remains in the region.

The double integral over $\int_{H_{x}^{\perp}}$ in Lemma 2 is thus asymptotic to a constant (numerically found to be approximately 0.02746 ) multiplied by $L^{-6}$ as $L \rightarrow \infty$. The extra factor of $L$ in Lemma 2 shows that

$$
F_{H}(t) \sim \frac{C_{d}}{t^{2} L^{5}}
$$

for some constant $C_{d}$ containing only geometrical factors, in the appropriate limit (first $t \rightarrow \infty$, then $L \rightarrow \infty$ ). Summing over $\mathbf{l} \in \hat{\mathbb{Z}}^{d-1}$ gives the Epstein zeta function $E\left(5 / 2 ; \mathbb{Z}^{d-1}\right) / \zeta(5)$. This is a convergent series for $d \leq 5$ and divergent for $d \geq 6$. Thus $d=6$ is a critical dimension for these incipient horizons. The divergence of the sum indicates that $F(t)$ has a slower decay with $t$ than $t^{-2}$ but a detailed calculation is required, 


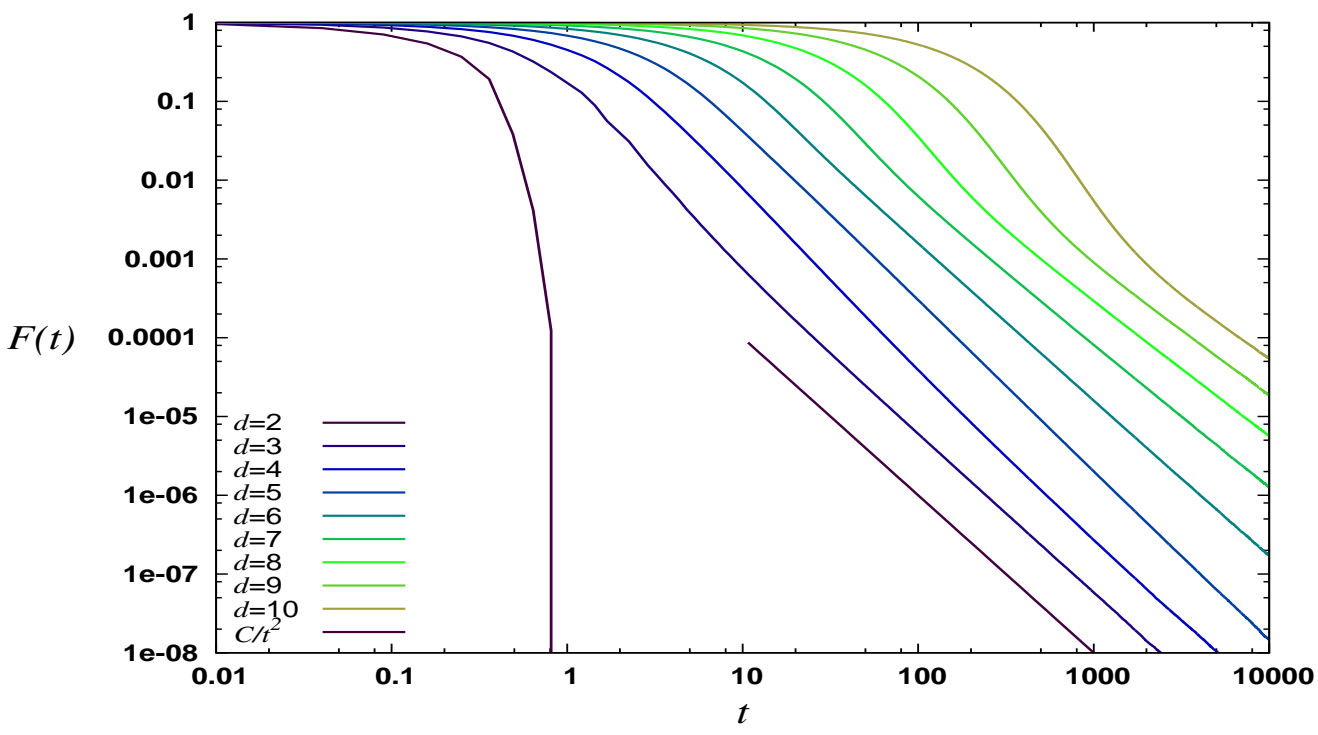

Figure 7: The free flight survival probability for dimensions $2 \leq d \leq 10$ for $r=0.5$ (touching) scatterers, showing escape at finite time for $d=2$, approach to $C / t^{2}$ for $2<d<5$ and slower decay for $d \geq 6$ as predicted by theory; fits for the relevant exponents are given in Tab. 2 .

\begin{tabular}{|c|cccccccc|}
\hline$d$ & 3 & 4 & 5 & 6 & 7 & 8 & 9 & 10 \\
\hline$n=10^{8}$ & 2.07 & 2.17 & 2.19 & 1.99 & 1.82 & 1.70 & 1.64 & 1.58 \\
$n=10^{9}$ & 2.02 & 2.20 & 2.17 & 1.98 & 1.80 & 1.67 & & \\
$n=10^{10}$ & 2.03 & 2.08 & 2.14 & 1.95 & & & & \\
\hline
\end{tabular}

Table 2: Numerical estimate of the exponent $\alpha$ from Conjecture 2 for cubic Lorentz gases with $r=1 / 2$, sample size $n$, and fitted for free flight survival probabilities $10^{2} / n<F(t)<10^{4} / n$.

taking into account the overlap of the relevant horizons, to yield a precise form. It is unlikely that the result would reach that of the non-incipient principal horizon, that is, $t^{-1}$; we propose Conj. 2 above.

Numerical tests of this conjecture are somewhat difficult due to the low packing fraction in high dimensions (so that individual trajectories survive for long times) and slow convergence to the long time limit. Scatterer radii $r=0.4$ and $r=0.6$ were discussed previously; see Figs. 3 and 4 . For a scatterer of radius 0.5 and dimension $d \geq 6$ the exponent itself is unknown; results are shown in Fig. 7 with fitted exponent in Tab. 2, and show exponents greater than 2 for low dimensions due to the finite times involved.

\section{Correlations and diffusion}

The above expression for $F(t)$ is also relevant to correlation functions, since conjecture 3 asserts that following a long flight, correlations decay very rapidly. For describing diffusion, we need velocity correlation functions. Figs. 8 and 9 show the velocity autocorrelation function (used below) compared with the sum of horizons predicted by Conj. 3 in the case of a three dimensional cubic Lorentz gas.

Recalling the discussion in previous sections, we are interested in the case of nonincipient principal horizons for which the function $F(t)$ decays as $C / t$; only in this case do we expect Eq. (8) to give superdiffusion, ie a nonzero value of $\mathcal{D}$. As discussed for $F(t)$, however, the coefficient is explicit. Now we give the proof of 


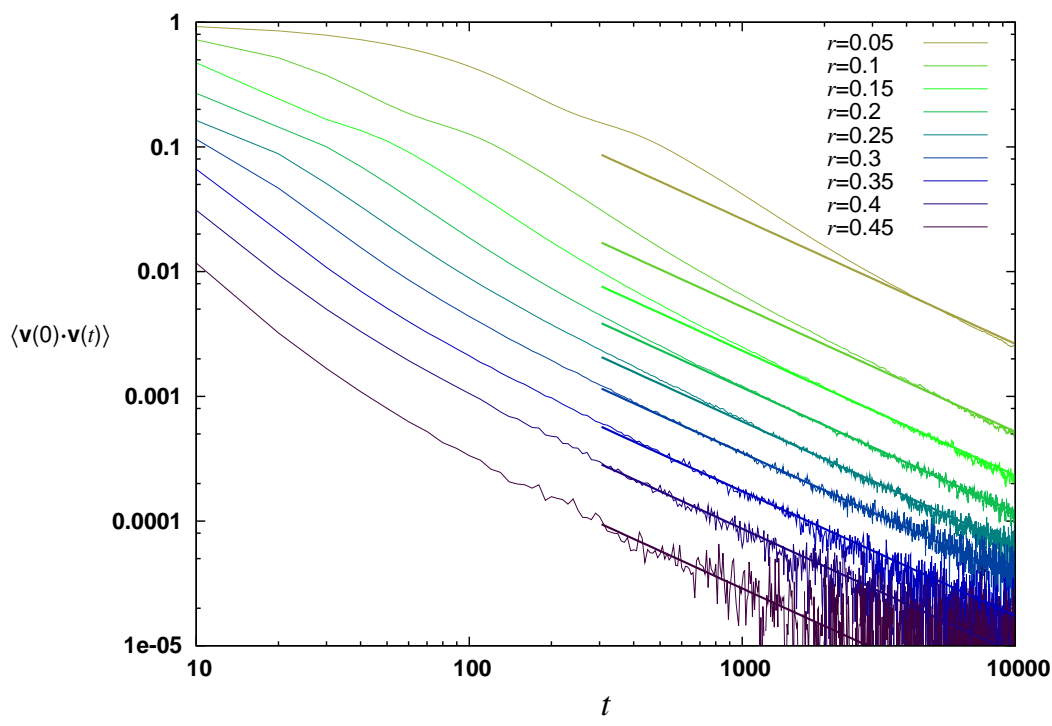

Figure 8: For $r<0.5$, Eq. 27 gives the limit of $t F(t)$ as $t \rightarrow \infty$ as a sum over horizons, assuming Conj. 1 . Conj. 3 applied to the components of the velocity asserts that the same limit is given by $t\left\langle\mathbf{v}_{0} \cdot \mathbf{v}_{t}\right\rangle$ related to the diffusion by Eq. (8). Here this correlation function is shown with a fit to the form $C_{r} / t$, for the three dimensional cubic Lorentz gas.

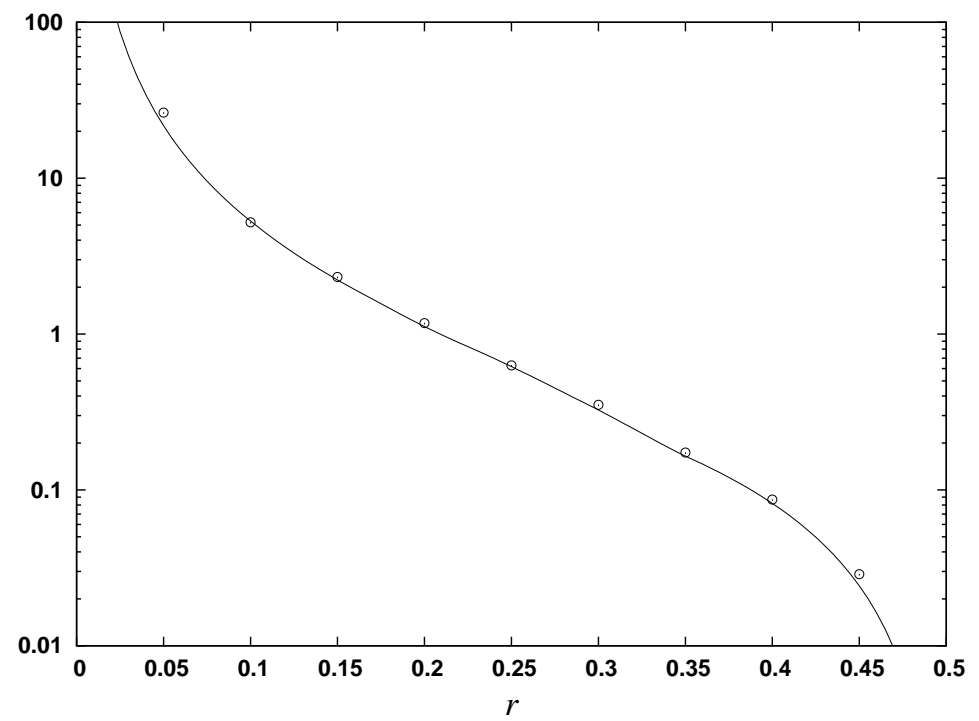

Figure 9: See Fig. 8 Comparison of the fitted $C_{r}$ (single points) with $\lim _{t \rightarrow \infty} t F(t)$ from Eq. 27 (smooth curve). 
Thm. 2

Proof. Assuming conjecture 3, the velocity correlations are determined to leading order by the integral over orbits that do not reach a scatterer in the given time, that is, $M_{t}$. For these orbits, $\mathbf{v}$ is a constant, so conjecture 1 can be applied. We then use the same method as in the proof of Lemma 2, except that the integrand varies significantly over the $\mathbf{v}^{\|}$space, ie $H_{v}$. Given that the perpendicular space is one-dimensional, we find a simple result in terms of the width of the horizon $w_{H}$ defined at the beginning of Sec. 6 .

$$
\left\langle v_{i}(0) v_{j}(t)\right\rangle \sim \sum_{H \in \mathcal{H}} \frac{w_{H}^{2} \int_{H_{v}} v_{i} v_{j}}{t S_{d-1} \mathcal{V}_{H}^{\perp}(1-\mathcal{P})}
$$

as $t \rightarrow \infty$. Now $H_{v}$ consists of all unit vectors $\mathbf{v}$ perpendicular to a unit vector $\mathbf{n}$, which depends on the horizon $H$ but we will suppress this in the notation. This $\mathbf{n}$ is parallel to a vector in $\mathcal{L}^{*}$ as discussed previously. Switching to a coordinate system based on $\mathbf{n}$ we can evaluate the integral to give

$$
\int_{H_{v}} v_{i} v_{j}=\left(\delta_{i j}-n_{i} n_{j}\right) V_{d-1}
$$

The cases $d>2$ and $d=2$ need to be derived separately, but give the same result. Putting this together we find the stated result.

For the case of the cubic lattice, the superdiffusion matrix is isotropic. For $1 /(2 \sqrt{2})<r<1 / 2$ we have only $d$ horizons of the form $(1,0,0, \ldots)$ and find that $\mathcal{D}_{i j}=\mathcal{D} \delta_{i j}$ with

$$
\mathcal{D}=\frac{(1-2 r)^{2}}{1-V_{d} r^{d}} \frac{\Gamma(d / 2)}{\sqrt{\pi} \Gamma((d-1) / 2)}
$$

while for $1 /(2 \sqrt{3})<r<1 /(2 \sqrt{2})$ there is an additional term coming from the $d(d-1)$ horizons of the form $(1, \pm 1,0, \ldots)$, which is thus larger by a factor of order $d$. In general this means that the large $d$ asymptotics at fixed $r$ has a different form every time a new horizon is introduced.

For the limit of small scatterers and fixed $d$ the sum over horizons is exactly the same as for $F(t)$ in Sec. 6. and we can directly combine Eqs. 19:31) to get

$$
\mathcal{D}=\frac{\pi^{(d-1) / 2}}{2^{d} d^{2} \Gamma((d+3) / 2) \zeta(d) r^{d-1}}+O\left(r^{1 / 2-\delta}\right)
$$

for $\delta>0$. Again it appears that this statement is equivalent to the Riemann Hypothesis under the same caveat (convergence of a sequence of contour integrals) as in Sec. 6 .

We can also compute the diffusion numerically, however even for the lowest dimensions, normal diffusion unrelated to the horizons dominates at accessible timescales; see Fig. 10.

\section{Diffusion in discrete time}

In order to compare our explicit expressions with other literature, we note that [45] uses exclusively discrete time properties (using $n$ rather than $t$ in the definitions), while [5, 11] link discrete to continuous time preperties. Recall the discussion in Sec. $22 \mathcal{D}^{\text {disc }}$ is formally infinite, however we can discuss the covariance of the limiting distribution, which is related to the superdiffusion coefficient by $\Xi=\mathcal{D}$ and to its discrete time version by $\Xi^{\text {disc }}=\tau \Xi$. The mean free time $\tau$ is given by 8

$$
\tau=\frac{1-\mathcal{P}}{|\partial \Omega \cap E|} \frac{S_{d-1}}{V_{d-1}}
$$

Here $|\partial \Omega \cap E|$ is the measure of the boundary of the scatterers, equal to $S_{d-1} r^{d-1}$ in the case of a single scatterer per unit cell, without overlapping. Thus in the latter case we find

$$
\tau=\frac{1-V_{d} r^{d}}{V_{d-1} r^{d-1}}
$$




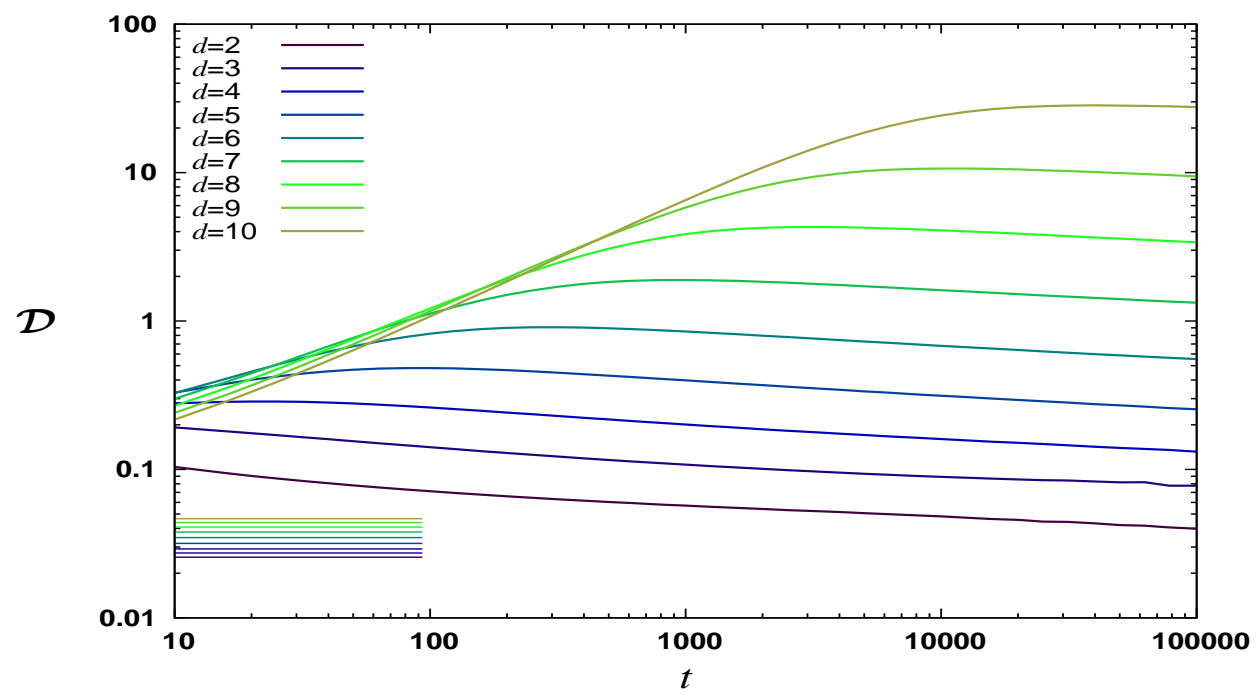

Figure 10: The superdiffusion coefficient $\mathcal{D}$ numerically (dotted lines) and theoretically (horizontal lines). At accessible timescales normal diffusion processes dominate.

which when combined with Thm. 2 and the above relations gives a simpler prefactor:

$$
\Xi_{i j}^{\mathrm{disc}}=\frac{1}{r^{d-1} S_{d-1}} \sum_{H \in \mathcal{H}} \frac{w_{H}^{2}}{\mathcal{V}_{H}^{\perp}}\left(\delta_{i j}-n_{i}(H) n_{j}(H)\right) .
$$

\section{Acknowledgements}

The author thanks Domokos Szász for suggesting the problem of diffusion in high dimensional Lorentz gases and for helpful discussions; acknowledges funding from the Royal Society and the organisers of "Chaotic and Transport Properties of Higher-Dimensional Dynamical Systems" (Cuernavaca, Jan 2011), where some ideas were further developed; and is also grateful to Leonid Bunimovich, Nikolai Chernov, Orestis Georgiou, Jens Marklof, Ian Melbourne, Zeev Rudnick, David Sanders and Trevor Wooley for helpful discussions.

\section{References}

[1] R. C. Baker, Acta Arith. 142, 267-302 (2010).

[2] P. Bálint and S. Gouëzel, Commun. Math. Phys. 263 461-512 (2006).

[3] P. Bálint and I. Melbourne, J. Stat. Phys. 133, 435-447 (2008).

[4] P. Bálint and I. P. Tóth, "Example for Exponential Growth of Complexity in a Finite Horizon Multidimensional Dispersing Billiard" (in preparation).

[5] P. M. Bleher, J. Stat. Phys. 66, 315-373 (1992).

[6] L. A. Bunimovich and C. P. Dettmann, Phys. Rev. Lett. 94, 100201 (2005). 
[7] L. A. Bunimovich and Ya. G. Sinai, Commun. Math. Phys. 78, 479-497 (1981).

[8] N. I. Chernov, J. Stat. Phys. 88, 1-29 (1997).

[9] N. I. Chernov, J. Stat. Phys. 127, 21-50 (2007).

[10] N. I. Chernov, private communication.

[11] N. I. Chernov and D. Dolgopyat, Russ. Math. Surv. 64, 651-699 (2009).

[12] N. I. Chernov and D. Dolgopyat, J. Amer. math. Soc. 22, 821-858 (2009).

[13] N. I. Chernov and R. Markarian, "Chaotic billiards" (Amer. Math. Soc., 2006).

[14] J. B. Conrey, Not. Amer. Math. Soc. 50, 341-353 (2003).

[15] J.-P. Conze, Ergod. Th. Dyn. Sys. 19, 1233-1245 (1999).

[16] M. Courbage, M. Edelman, S. M. Saberi Fathi and G. M. Zaslavsky Phys. Rev. E 77, 036203 (2008).

[17] C. P. Dettmann, "The Lorentz gas as a paradigm for nonequilibrium stationary states," in "hard ball systems and the Lorentz gas" (Ed. D. Szász) Encyclopaedia of Mathematical Sciences 101, 315-365 (2000).

[18] D. Dolgopyat, D. Szász and T. Varjú, Duke Math. J. 148, 459-499 (2009).

[19] B. Friedman and R. F. Martin Jr., Phys. Lett. 105A, 23-26 (1984).

[20] H. Fujisaka and S. Grossman, Z. Phys. B. 48, 261-275 (1982).

[21] F. Galton, "Natural inheritance" (Macmillan, New York, 1894).

[22] P. Gaspard, J. Stat. Phys. 68, 673-747 (1992).

[23] T. Gilbert, H. C. Nguyen and D. P. Sanders, J. Phys. A: Math. Theor. 44, 065001 (2011).

[24] T. Harayama and P. Gaspard, Phys. Rev. E 64, 036215 (2001).

[25] T. Harayama, R. Klages and P. Gaspard, Phys. Rev. E 66, 026211 (2002).

[26] G. Keller, P. Howard and R. Klages, Nonlinearity 21, 1719 (2008).

[27] R. Klages and C. Dellago, J. Stat. Phys. 101, 145-159 (2000).

[28] R. Klages and N. Korabel, J. Phys. A 35, 4823-4836 (2002).

[29] H. A. Lorentz, Proc. Amst. Acad. 7, 438-453 (1905).

[30] J. Marklof and A. Strömbergsson, Ann. Math. 174, 225-298 (2011).

[31] J. Marklof and A. Strömbergsson, Geom. \& Func. Anal. 21, 560-647 (2011).

[32] H. Matsuoka and R. F. Martin, J. Stat. Phys. 88 81-103 (1997).

[33] I. Melbourne, Proc. London Math. Soc. 98 163-190 (2009).

[34] I. Melbourne, private communication.

[35] I. Melbourne and A. Török, "Convergence of moments for Axiom A and non-uniformly hyperbolic flows" Ergod. Th. \& Dyn. Sys. (to appear).

[36] P. Nándori, D. Szász and T. Varjú, in preparation. 
[37] D. Sanders, Phys. Rev. E 78160101 (2008).

[38] P. Sarnak and A. Strömbergsson, Invent. Math. 165, 115-151 (2006).

[39] M. Schell, S. Fraser and R. Kapral, Phys. Rev. A 26, 504-521 (1982).

[40] K. Schmidt, C. R. Acad. Sci. Paris, Ser I 327, 837-842 (1998).

[41] D. Schumayer and D. A. Hutchinson, Rev. Mod. Phys. 83, 307-330 (2011).

[42] Ya. G. Sinai, Russ. Math. Surv. 25, 137-189 (1970).

[43] J. Steuding, Math. Ann. 333, 689-697 (2005).

[44] D. Szász, Nonlinearity 21, T187-T193 (2008).

[45] D. Szász and T. Varjú, J. Stat. Phys. 129, 59-80 (2007).

[46] A. Zacherl, T. Geisel, J. Nierwetberg and G. Radons, Phys. Lett. 114A, 317-321 (1986). 